\title{
CONSIDERATION OF THE UNCERTAINTY IN DIMENSIONING OF A GEARBOX OF A WIND TURBINE
}

\author{
Hassen Trabelsi, Amir Guizani, Maher Barkallah \\ Laboratory of Mechanics, Modelling and Production (LA2MP), National School of Engineering of Sfax, \\ University of Sfax, Tunisia \\ e-mail: amir.guizani@live.fr \\ Moncef Hammadi \\ Quartz EA7393, Supméca-Paris, Saint-Ouen, France \\ Ayman Haddrich, Mohamed Haddar \\ Laboratory of Mechanics, Modelling and Production (LA2MP), National School of Engineering of Sfax, \\ University of Sfax, Tunisia
}

\begin{abstract}
The paper deals with the design approach of a subdefinite mechatronic system and focuses on the sizing stage of a gearbox of a wind turbine based on the interval computation method. Indeed, gearbox design variables are expressed by intervals to take into account the uncertainty in the estimation of these parameters. The application of the interval computation method allows minimizing the number of simulations and enables obtaining a set of solutions instead of a single one. The dynamic behavior of the gearbox is obtained using the finite element method. The challenge here is to get convergent results with intervals that reflect the efficiency of the applied method. Thus, several mathematical formulations have been tested in static study and evaluated in the case of a truss. Then the interval computation method was used to simulate the behavior of the wind turbine gearbox.
\end{abstract}

Keywords: uncertainty, interval computation, Finite Element Method, truss, dynamic behavior, gearbox

\section{Introduction}

Mechatronics is one of the most dynamically developing fields of technology and science. In fact, mechatronics refers to systems which integrate mechanical and electrical components linked through a control system and information technology (Dieterle, 2005; Habib, 2007; Karnopp et $a l ., 2012)$. The preliminary design of sub-defined mechatronic systems is upstream of the designing process (Hamza et al., 2015; Amendola et al., 2017). Indeed, from a set of requirements and constraints, it consists in determining a set of possible solutions that respect the required performances in a very large research area (Hammadi et al., 2012; Guizani et al., 2014). This choice is structured by a partial and uncertain knowledge of the future system and its environment. The conventional design approaches used to study the dynamic behavior of a mechatronic system are based on a repetitive loop type "dimensioning-modeling and simulation - return to the initial stage in case of divergence or failure" (Alefeld and Mayer, 2000; Trabelsi et al., 2012). These sizing techniques appear obsolete. Moreover, these classic approaches are often expensive and require higher computation times in order to obtain the optimal values of design parameters (Martins and Lambe, 2013). In recent years, several methods have been developed to treat this kind of problems (Hughes, 2012; Ma et al., 2013) and, more precisely, in systems in which equations of motions are defined by partial differential equations. Among the methods used to solve this type of equations, we cite the finite element method (FEM) (Hughes, 2012; Zienkiewicz 
and Taylor, 2005). In order to consider uncertainty in some design parameters of the system to be designed, the FEM associated with the interval calculation method was used to model and simulate its dynamic behavior with uncertainty (Gilewski et al., 2015; Fang et al., 2015).

In the works cited previously, the coupling between the interval computation and FEM provided generally disappointing results and often divergent (Ma et al., 2013, Gilewski et al., 2015). Indeed, the area of the obtained solutions increases when the simulation moves away from the starting points. Therefore, the aim of this paper is to find a solution, which makes the use of the interval computation with FEM possible in the dynamic study. For that, we started with static sizing of a truss as a simple example. Many series of simulations were done in order to reduce the divergence of the results and to choose an optimal mathematical formulation. Then, we chose as a mechatronic system the gearbox of a wind turbine to study its dynamic behavior. The main idea of this work is to simulate with bounded values describing behavior of the system, contrary to the conventional method that is based on a fixed value.

In fact, the bounded values in the case of a truss are useful for a material that presents lightly different proprieties or for composite materials whose properties are inconstant. In the case of a gearbox, the challenge is to obtain convergent results that reflect the efficiency of the use of the interval computation technique coupled with FEM.

The remaining of this paper is organized as follows. First, the problem of static sizing of a truss using FEM coupled with the interval computation method is presented in Section 2. Then, in Section 3, the previously mentioned method is applied to the case of the gearbox system of a wind turbine to obtain the dynamic behavior with uncertainty. Finally, some conclusions are drawn based on the obtained results in Section 4.

\section{Static simulation of a truss with uncertainty}

The objective of this part is to evaluate the interval computation method to statically simulate systems that present uncertainties in their design parameters, and in which their behavior models are defined by partial differential equations.

The chosen truss presents a well-known example in metal structures. It is composed of two perpendicular similar structures and contains nine nodes articulated and fourteen bars. It is illustrated in Fig. 1. The structure is subjected to a vertical point load $F_{r}=100 \mathrm{kN}$ applied at node number three. The boundary conditions taken into account are support bearings at nodes one and seven.

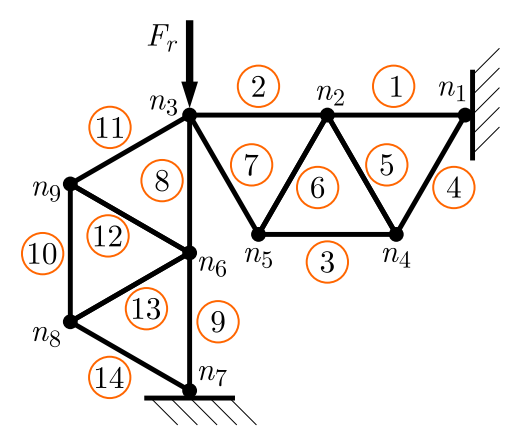

Fig. 1. Truss

After characterization of the structure, the discretization with FEM is done in the next Section. The values of different parameters used in the studied model are given in Table 1. 
Table 1. Characteristics of the truss

\begin{tabular}{|l|l|c|c|}
\hline \multicolumn{1}{|c|}{ Variables } & \multicolumn{1}{|c|}{ Nomenclature } & Values & Units \\
\hline \hline$E$ & Young's modulus & $2 \cdot 10^{11}$ & $\mathrm{~N} / \mathrm{m}^{2}$ \\
\hline$S$ & section & 0.01 & $\mathrm{~m}^{2}$ \\
\hline$L_{1}=L_{2}=L_{3}=L_{8}=L_{9}=L_{10}$ & length of bars $1,2,3,8,9$ and 10 & 10 & $\mathrm{~m}$ \\
\hline $\begin{array}{c}L_{4}=L_{5}=L_{6}=L_{7}=L_{11} \\
=L_{12}=L_{14}=L_{15}\end{array}$ & $\begin{array}{l}\text { length of bars } 4,5,6,7,11,12,14 \\
\text { and } 15\end{array}$ & $10 \sqrt{2}$ & $\mathrm{~m}$ \\
\hline
\end{tabular}

\subsection{Finite Element Method: elementary element}

The truss is discretized with dabble-node elements with two degrees of freedom. Therefore, the components of the displacement vector $\mathbf{u}$ are written as follows: $\mathbf{u}=\left[x_{1}, y_{1}, x_{2}, y_{2}\right]^{\mathrm{T}}$.

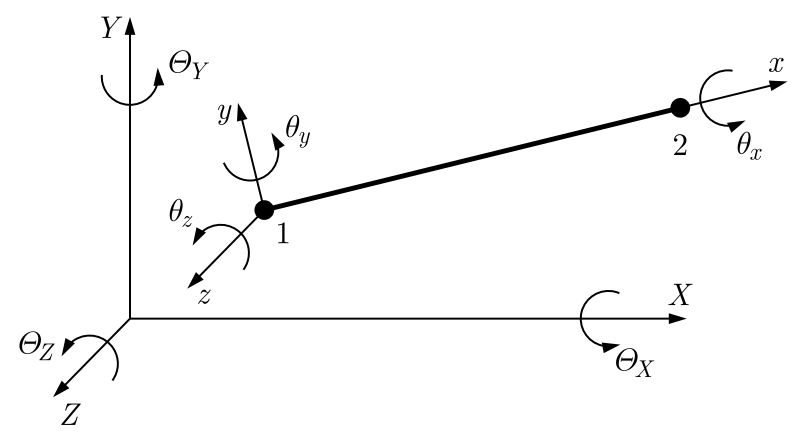

Fig. 2. Two-node element

The stiffness matrix is expressed by

$$
\mathbf{K}_{p}=\left[\begin{array}{cccc}
s_{1} & 0 & -s_{1} & 0 \\
0 & 0 & 0 & 0 \\
-s_{1} & 0 & s_{1} & 0 \\
0 & 0 & 0 & 0
\end{array}\right] \quad s_{1}=\frac{E S}{L}
$$

In addition, $\mathbf{T}$ is the transformation matrix

$$
\mathbf{T}=\left[\begin{array}{cccc}
\cos \theta & \sin \theta & 0 & 0 \\
-\sin \theta & \cos \theta & 0 & 0 \\
0 & 0 & \cos \theta & \sin \theta \\
0 & 0 & -\sin \theta & \cos \theta
\end{array}\right]
$$

After definition of the elementary matrix of different parts of the structure, the chosen model is detailed in the next part.

\subsection{Calculation of unknown displacements}

The unknown displacements are solutions to the equation

$$
\mathbf{K X}=\mathbf{F}
$$

where $\mathbf{K}$ is the stiffness matrix, $\mathbf{X}$ is the unknown displacement vector and $\mathbf{F}$ is the nodal forces vector

$$
\begin{aligned}
& \mathbf{F}=\left[0,0,0,0,0, F_{r}, 0,0,0,0,0,0,0,0,0,0,0,0\right]^{\mathrm{T}} \\
& \mathbf{X}=\left[x_{1}, y_{1}, x_{2}, y_{2}, x_{3}, y_{3}, x_{4}, y_{4}, x_{5}, y_{5}, x_{6}, y_{6}, x_{7}, y_{7}, x_{8}, y_{8}, x_{9}, y_{9}\right]^{\mathrm{T}}
\end{aligned}
$$


The system is evaluated by three mathematical formulations which were developed by Gauss, Krawski and Hansen (Nirmala et al., 2013; Hansen and Sengupta, 1981; Neumaier, 1999). To avoid possible rounding errors and divergent results, we propose to use an intersection method. It consists in taking an intersection between three intervals generated by the previously mentioned methods. The idea is to obtain solution intervals as small as possible, as shown in Fig. 3.

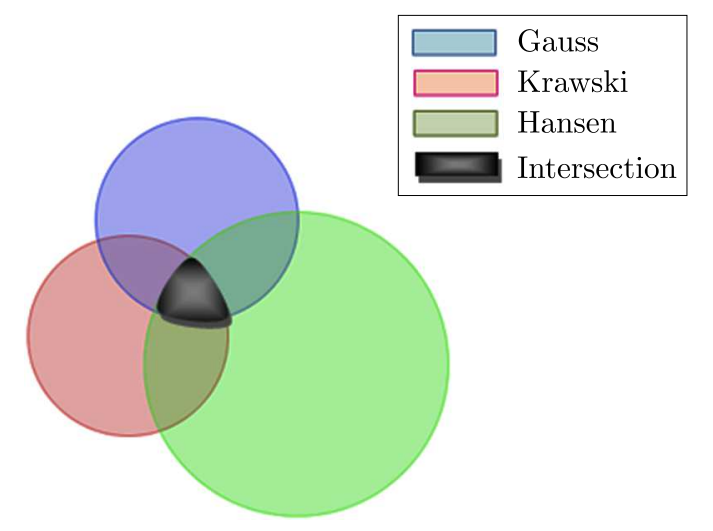

Fig. 3. Enclosed set for three different methods

Therefore, different formulations are tested in order to compare the accuracy of the methods and to prove validity of the intersection method.

\subsection{Results of interval computation}

The calculated displacements with a fixed value of Young's modulus $E$ are presented in the following vector $\mathbf{X}=[0,0,0.0151,-0.4916,-0.0000,-1.4775,0.2962,-0.2534,0.3265$, $-0.7602,0.2034,-0.7388,0,0,-0.2677,-0.6157,-0.2677,-0.8618]$.

The displacement vector values calculated with the fixed value of $E$ will be useful to make a comparison with the values obtained by calculation with intervals. Thus, uncertainty of different percentages (from $0.2 \%$ to $2 \%$ ) is introduced into Young's modulus $E$ in order to test various methods mentioned previously. To correctly interpret the results obtained in Table 2, the displacements of nodes number three and eight are plotted in both $x$ and $y$ directions as a function of the percentage of uncertainty.

Through Fig. 4, we note that the envelopes obtained with the methods of Hansen and the intersection are closer to the displacement of nodes number three and eight simulated with fixed values. Indeed, the framing envelope given by these two methods is less thick compared to the method of Gauss and Krawski. The efficient framing is given by the intersection method since it provides the smallest areas.

In the next Section, these methods are applied in the case of dynamic simulation of a gearbox system of a wind turbine.

\section{Gearbox system of a wind turbine}

The gearbox of a wind turbine is a system to reduce the motor rotation speed while increasing its torque. The model of the system is composed of three components: the motor, reducer and transmission part (Chaari et al., 2016). The transmission part comprises four elements: the drive and driven shaft, pinion and wheel, as shown in Fig. 5.

The studied gearbox has parallel axes composed of two cylindrical wheels with straight teeth. The different dimensions of the analyzed example are mentioned in Table 3. 
Table 2. Values of displacements with uncertainties

\begin{tabular}{|c|c|c|c|c|c|}
\hline & $\begin{array}{l}\text { Uncer- } \\
\text { tainty }\end{array}$ & Gauss & Krawski & Hansen & Intersection \\
\hline \multirow{7}{*}{$\begin{array}{c}x_{3} \\
{[\mathrm{~mm}]}\end{array}$} & $0 \%$ & 0 & 0 & 0 & 0 \\
\hline & $0.2 \%$ & {$[-0.1680,0.1567]$} & {$[-0.0346,0.0346]$} & {$[-0.0338,0.0338]$} & {$[-0.0338,0.0338]$} \\
\hline & $0.8 \%$ & {$[-1.1411,0.8518]$} & {$[-0.3045,0.3045]$} & {$[-0.1817,0.1817]$} & {$[-0.1817,0.1817]$} \\
\hline & $1.2 \%$ & {$[-3.9941,2.7392]$} & {$[-0.8339,0.8339]$} & {$[-0.3489,0.3489]$} & {$[-0.3489,0.3489]$} \\
\hline & $1.4 \%$ & {$[-13.8887,8.9001]$} & {$[-0.9225,0.9225]$} & {$[-0.4709,0.4709]$} & {$[-0.4709,0.4709]$} \\
\hline & $1.6 \%$ & $N a N$ & {$[-1.0022,1.0022]$} & {$[-0.6354,0.6354]$} & {$[-0.6354,0.6354]$} \\
\hline & $2 \%$ & $N a N$ & $N a N$ & $N a N$ & $N a N$ \\
\hline \multirow{7}{*}{$\begin{array}{c}y_{3} \\
{[\mathrm{~mm}]}\end{array}$} & $0 \%$ & -1.4775 & -1.4775 & -1.4775 & -1.4775 \\
\hline & $0.2 \%$ & {$[-1.6522,-1.3221]$} & {$[-1.5132,-1.4418]$} & {$[-1.5129,-1.4428]$} & {$[-1.5129,-1.4428]$} \\
\hline & $0.8 \%$ & {$[-2.6260,-0.6870]$} & {$[-1.6919,-1.2631]$} & {$[-1.6436,-1.3231]$} & {$[-1.6436,-1.3231]$} \\
\hline & $1.2 \%$ & {$[-5.2967,0.9913]$} & {$[-1.5190,-1.0174]$} & {$[-1.7644,-1.2224]$} & {$[-1.5190,-1.2224]$} \\
\hline & $1.4 \%$ & {$[-14.2120,6.6733]$} & {$[-1.4402,-0.9685]$} & {$[-1.8425,-1.1614]$} & {$[-1.4402,-1.1614]$} \\
\hline & $1.6 \%$ & $N a N$ & {$[-1.3692,-0.9245]$} & {$[-1.9397,-1.0892]$} & {$[-1.3692,-0.9245]$} \\
\hline & $2 \%$ & $N a N$ & $N a N$ & $N a N$ & $N a N$ \\
\hline \multirow{7}{*}{$\begin{array}{c}x_{8} \\
{[\mathrm{~mm}]}\end{array}$} & $0 \%$ & -0.2677 & -0.2677 & -0.2677 & -0.2677 \\
\hline & $0.2 \%$ & {$[-0.3535,-0.1920]$} & {$[-0.2906,-0.2447]$} & {$[-0.2902,-0.2453]$} & {$[-0.2902,-0.2453]$} \\
\hline & $0.8 \%$ & {$[-0.8693,0.1295]$} & {$[-0.4393,-0.0960]$} & {$[-0.3817,-0.1571]$} & {$[-0.3817,-0.1571]$} \\
\hline & $1.2 \%$ & {$[-2.2916,0.9755]$} & {$[-0.7138,0.1785]$} & {$[-0.4769,-0.0687]$} & {$[-0.4769,-0.0687]$} \\
\hline & $1.4 \%$ & {$[-7.1082,3.7166]$} & {$[-0.7612,0.2259]$} & {$[-0.5431,-0.0085]$} & {$[-0.5431,-0.0085]$} \\
\hline & $1.6 \%$ & $N a N$ & {$[-0.8039,0.2686]$} & {$[-0.6299,0.0740]$} & {$[-0.6299,0.0740]$} \\
\hline & $2 \%$ & $N a N$ & $N a N$ & $N a N$ & $N a N$ \\
\hline \multirow{7}{*}{$\begin{array}{c}y_{8} \\
{[\mathrm{~mm}]}\end{array}$} & $0 \%$ & -0.6157 & -0.6157 & -0.6157 & -0.6157 \\
\hline & $0.2 \%$ & {$[-0.7218,-0.5291]$} & {$[-0.6455,-0.5859]$} & {$[-0.6452,-0.5867]$} & {$[-0.6452,-0.5867]$} \\
\hline & $0.8 \%$ & {$[-1.3550,-0.2696]$} & {$[-0.8040,-0.4274]$} & {$[-0.7567,-0.4839]$} & {$[-0.7567,-0.4839]$} \\
\hline & $1.2 \%$ & {$[-3.0489,0.1556]$} & {$[-1.0569,-0.1745]$} & {$[-0.8625,-0.3941]$} & {$[-0.8625,-0.3941]$} \\
\hline & $1.4 \%$ & {$[-8.6827,1.2956]$} & {$[-1.1037,-0.1277]$} & {$[-0.9320,-0.3382]$} & {$[-0.9320,-0.3382]$} \\
\hline & $1.6 \%$ & $N a N$ & {$[-1.1460,-0.0855]$} & {$[-1.0192,-0.2707]$} & {$[-1.0192,-0.0855]$} \\
\hline & $2 \%$ & $N a N$ & $N a N$ & $N a N$ & $N a N$ \\
\hline
\end{tabular}

\subsection{Dynamic analysis of the gearbox system of the wind turbine}

To obtain a dynamic model of the gearbox, the two shafts are discretized with two-node elements with four degrees of freedom. Therefore, components of the vector $\mathbf{u}$ are written as following

$$
\mathbf{u}=\left[x_{1}, y_{1}, \theta_{x 1}, \theta_{y 1}, x_{2}, y_{2}, \theta_{x 2}, \theta_{y 2}\right]^{\mathrm{T}}
$$

The values of the different parameters used in the gearbox model are given in Table 3.

The contact between the pinion and the wheel is expressed as follows

$$
\mathbf{M}_{c}\left[\begin{array}{c}
\ddot{\theta}_{1} \\
\ddot{\theta}_{2}
\end{array}\right]+\mathbf{K}_{c}\left[\begin{array}{c}
\theta_{1} \\
\theta_{2}
\end{array}\right]=\left[\begin{array}{l}
C_{1} \\
C_{2}
\end{array}\right]
$$

with

$$
\mathbf{M}_{c}=\left[\begin{array}{cc}
I_{1} & 0 \\
0 & I_{2}
\end{array}\right] \quad \mathbf{K}_{c}=\left[\begin{array}{ll}
s_{10} K(t) & s_{12} K(t) \\
s_{21} K(t) & s_{20} K(t)
\end{array}\right]
$$


(a)

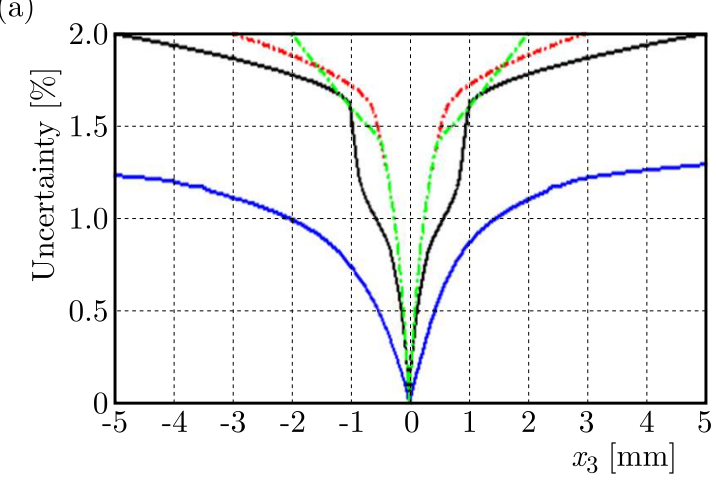

(c)

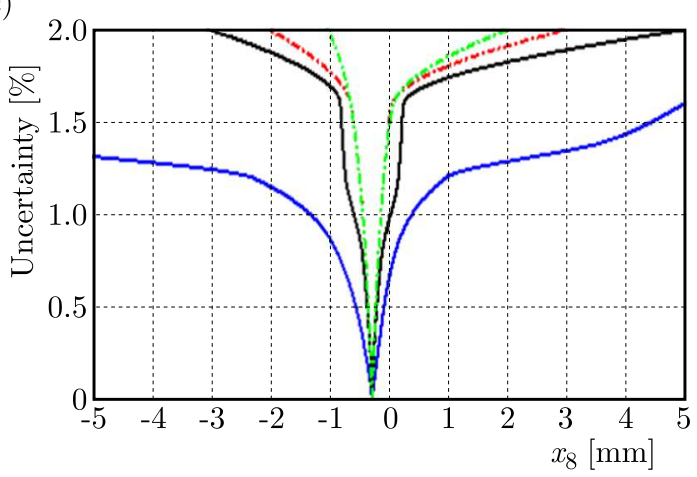

(b)

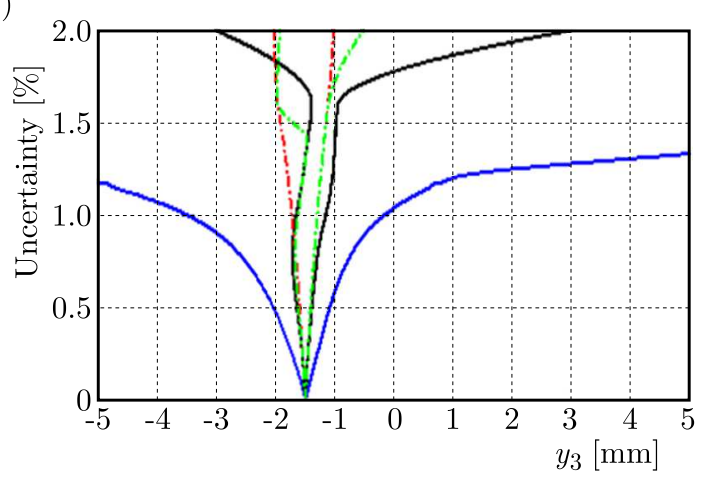

(d)

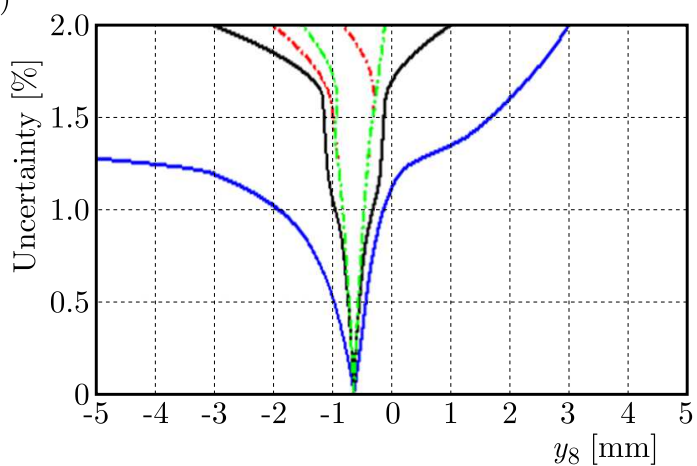

\begin{tabular}{lll}
\hline - Gauss & -.-... Hansen \\
— Krawski & -..... Intersection \\
\hline
\end{tabular}

Fig. 4. Values of displacements with uncertainty

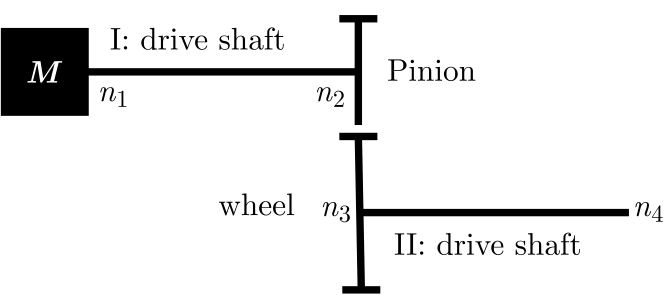

Fig. 5. Model of the gearbox reducer system of a wind turbine

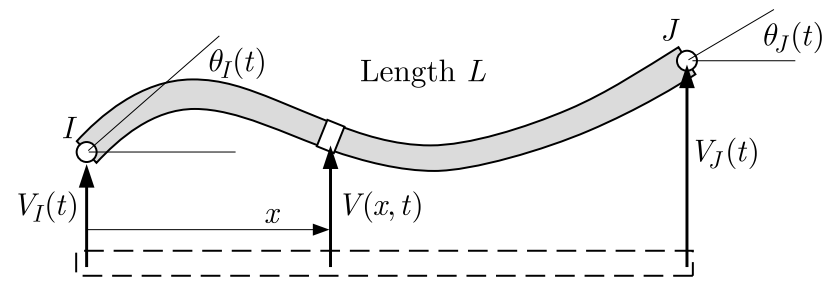

Fig. 6. Discretization

and

$$
I_{1}=\frac{1}{2} M_{1}\left(\frac{d_{1}}{2}\right)^{2} \quad I_{2}=\frac{1}{2} M_{2}\left(\frac{d_{2}}{2}\right)^{2}
$$

The mesh stiffness is a function of time, the average part of $\phi$ is removed

$$
K(t)=K_{12}\left(1+\frac{K_{12}-k_{12}}{K_{12}}\right)[\phi-\operatorname{mean}(\phi)] \quad \phi=\operatorname{square}\left[2 \pi f_{\text {en }} t\left(c_{12}-1\right) 100\right]
$$


Table 3. Characteristics of the gearbox system

\begin{tabular}{|c|l|c|c|}
\hline Variables & \multicolumn{1}{|c|}{ Nomenclature } & Values & Units \\
\hline \hline$Z_{1}$ & number of sprocket (pinion) teeth & 20 & - \\
\hline$Z_{2}$ & number of teeth of the wheel & 40 & - \\
\hline$m$ & module & 0.003 & $\mathrm{~m}$ \\
\hline$b$ & width of teeth & 0.030 & $\mathrm{~m}$ \\
\hline$d_{i 1}$ & inside diameter of pinion & 0.020 & $\mathrm{~m}$ \\
\hline$d_{i 2}$ & inside diameter of wheel & 0.030 & $\mathrm{~m}$ \\
\hline$N_{1}$ & rotation speed of pinion & 1200 & $\mathrm{tr} / \mathrm{min}$ \\
\hline$N_{2}$ & rotation speed of wheel & 750 & $\mathrm{tr} / \mathrm{min}$ \\
\hline$A$ & pressure angle & 20 & $\mathrm{deg}$ \\
\hline$r_{o}$ & density of steel & 7850 & $\mathrm{~kg} / \mathrm{m}^{3}$ \\
\hline$C_{1}$ & motor torque & 50 & $\mathrm{Nm}$ \\
\hline$C_{2}$ & receiver torque & 100 & $\mathrm{Nm}$ \\
\hline$M_{1}$ & mass of the pinion & 1.8 & $\mathrm{Kg}$ \\
\hline$M_{2}$ & mass of the wheel & 2.5 & $\mathrm{Kg}$ \\
\hline$f_{e n}$ & gear mesh frequency & $Z_{1} N_{1} / 60$ & $\mathrm{~Hz}$ \\
\hline$T$ & gear mesh period & $1 / f$ & $\mathrm{~s}$ \\
\hline$L$ & length of shafts & 0.048 & $\mathrm{~m}$ \\
\hline
\end{tabular}

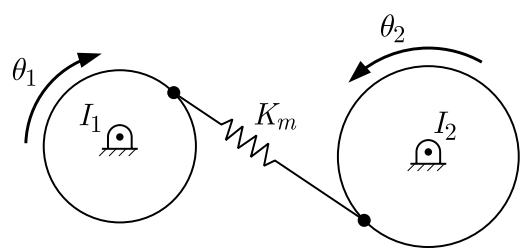

Fig. 7. Dynamic model of the transmission with damping

The mesh stiffness is given by

$$
K_{12}=c_{12} k_{12}
$$

where $c_{12}$ is the driving ratio of the transmission

$$
c_{12}=\frac{1+\frac{1}{Z_{1}}}{\pi \cos \alpha\left(\frac{\sin \alpha}{2}+\sqrt{\frac{(\sin \alpha)^{2}}{4}+\frac{2}{Z_{1}^{2}}}\right)}+\frac{1+\frac{1}{Z_{2}}}{\pi \cos \alpha\left(\frac{\sin \alpha}{2}+\sqrt{\frac{(\sin \alpha)^{2}}{4}+\frac{2}{Z_{2}^{2}}}\right)}
$$

and $k_{12}$ is stiffness of a pair of teeth in contact (Henriot, 1978)

$$
k_{12}=\frac{b}{0.04723+\frac{0.15551}{Z_{1}}+\frac{0.25791}{Z_{2}}}
$$

Finally, the damping matrix is given by

$$
\mathbf{C}=\alpha \mathbf{M}+\beta \mathbf{K}
$$

Therefore, the system model is calculated by the following equation

$$
\mathbf{M}_{t o t a l} \ddot{\mathbf{u}}+\mathbf{C}_{\text {total }} \dot{\mathbf{u}}+\mathbf{K}_{\text {total }} \mathbf{u}=\mathbf{F}(\mathbf{u})
$$


with

$$
\begin{aligned}
& M_{\text {total }}=M_{m}+M_{c}+M_{r} \quad C_{\text {total }}=C_{m}+C_{r} \quad K_{\text {total }}=K_{m}+K_{c}+K_{r} \\
& C_{m}=C_{r}=C \quad K_{m}=K_{r}=K
\end{aligned}
$$

Equation (4.10) is written as follows

$$
\begin{aligned}
& {\left[\begin{array}{cccc}
M_{m} & 0 & 0 & 0 \\
0 & M_{m}+M_{c} & 0 & 0 \\
0 & 0 & M_{r}+M_{c} & 0 \\
0 & 0 & 0 & M_{r}
\end{array}\right] \ddot{\mathbf{u}}+\left[\begin{array}{cccc}
C_{m} & 0 & 0 & 0 \\
0 & C_{m} & 0 & 0 \\
0 & 0 & C_{r} & 0 \\
0 & 0 & 0 & C_{r}
\end{array}\right] \dot{\mathbf{u}}} \\
& +\left[\begin{array}{ccccc}
K_{m} & 0 & 0 & 0 \\
0 & K_{m}+K_{c} & 0 & 0 \\
0 & 0 & K_{r}+K_{c} & 0 \\
0 & 0 & 0 & K_{r}
\end{array}\right] \mathbf{u}=\mathbf{F}(\mathbf{u})
\end{aligned}
$$

The mass matrix is expressed as follows

$$
\mathbf{M}=\left[\begin{array}{cccccccc}
78 & 0 & 0 & 22 \frac{L}{2} & 27 & 0 & 0 & -13 \frac{L}{2} \\
0 & 78 & -22 \frac{L}{2} & 0 & 0 & 27 & -13 \frac{L}{2} & 0 \\
0 & -22 \frac{L}{2} & 8\left(\frac{L}{2}\right)^{2} & 0 & 0 & -13 \frac{L}{2} & -6\left(\frac{L}{2}\right)^{2} & 0 \\
22 \frac{L}{2} & 0 & 0 & 8\left(\frac{L}{2}\right)^{2} & 13 \frac{L}{2} & 0 & 0 & -6\left(\frac{L}{2}\right)^{2} \\
27 & 0 & 0 & 13 \frac{L}{2} & 78 & 0 & 0 & 22 \frac{L}{2} \\
0 & 27 & -13 \frac{L}{2} & 0 & 0 & 78 & -22 \frac{L}{2} & 0 \\
0 & -13 \frac{L}{2} & -6\left(\frac{L}{2}\right)^{2} & 0 & 0 & -22 \frac{L}{2} & 8\left(\frac{L}{2}\right)^{2} & 0 \\
-13 \frac{L}{2} & 0 & 0 & -6\left(\frac{L}{2}\right)^{2} & 22 \frac{L}{2} & 0 & 0 & 8\left(\frac{L}{2}\right)^{2}
\end{array}\right]
$$

\subsection{Results of simulation}

Modeling and simulation were done using the Intlab interval calculator library in Matlab software. The computation process converges to the results shown in Figs. 8, 9, 10 and 11. Computation time takes $110 \mathrm{~s}$ on a regular computing machine (Intel (R) Core(TM) i7-6500U CPU@2.50GHz, 2 cores).

The evaluation of the Newmark method (Faroughi and Lee, 2015) with uncertainties in real cases provided disappointing and often divergent results. For this, the choice of design variables expressed by intervals acts on the convergence of the model. The objective of this part is to study the dynamic behavior of the gearbox of the wind turbine in the presence of uncertainties on some design variables. The main advantage is to obtain convergent results that reflect the efficiency of the interval calculation technique coupled with the Newmark method to study the dynamic behavior of the system.

The design parameters studied with uncertainties are the module $m$ and the receiver torque $c_{r}$. Indeed, the uncertainty on $m$ reflects in real cases the manufacturing problems and the precision error of diameters of the gear. Whereas, the uncertainty on $c_{r}$ is introduced since the estimation of the receiving torque is imprecise. For this, we introduced $3 \%$ of uncertainty on $m$ and $c_{r}$. Figures 8, 9, 10 and 11 show eight displacements of the vector $\mathbf{u}$. As shown in Figs. 8 and 9, the variation of the module $m$ has a great influence on the rotational movements 


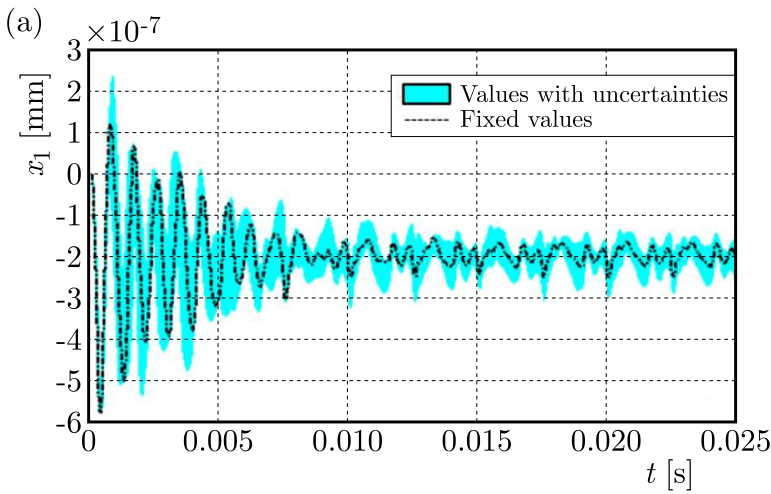

(b)
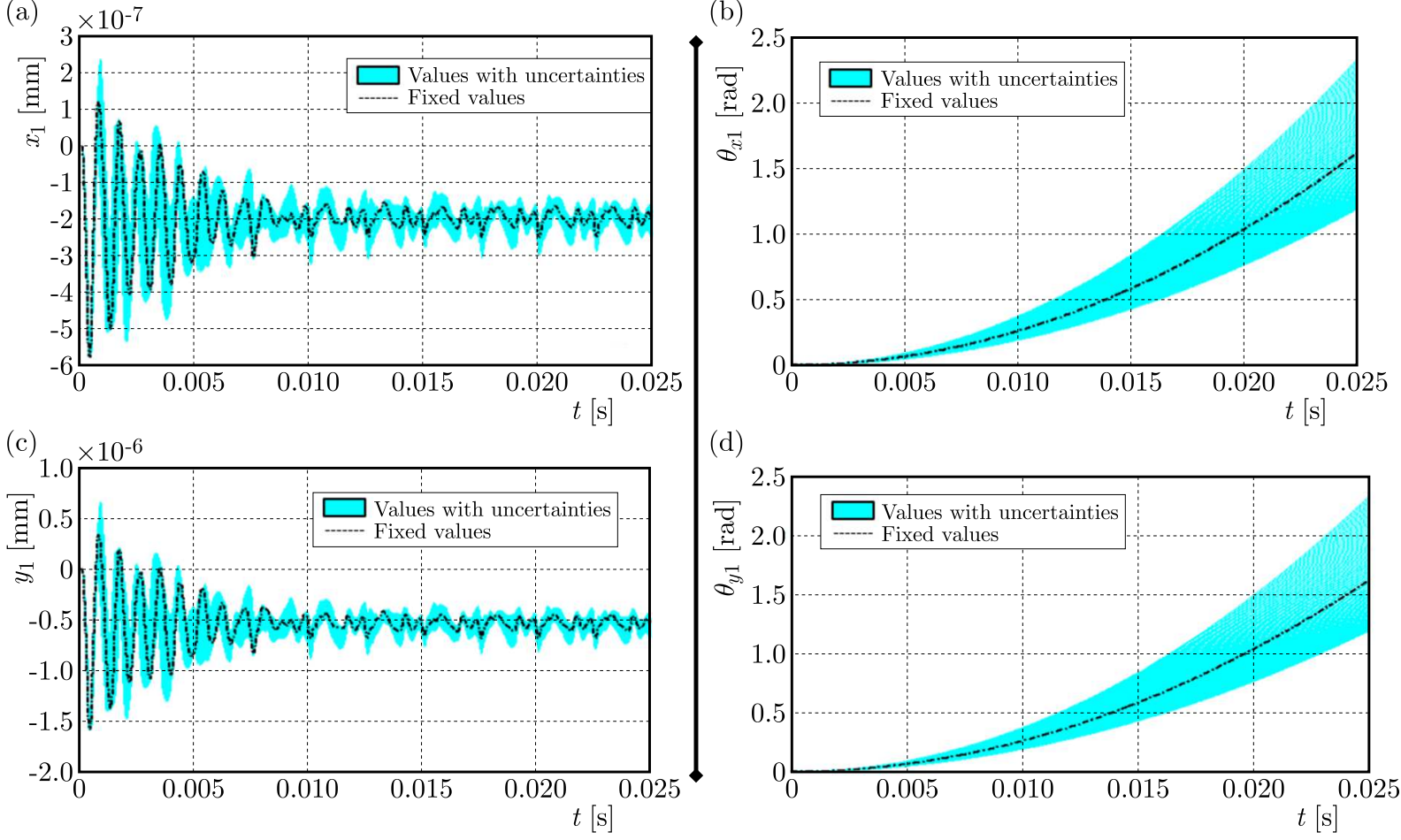

(d)

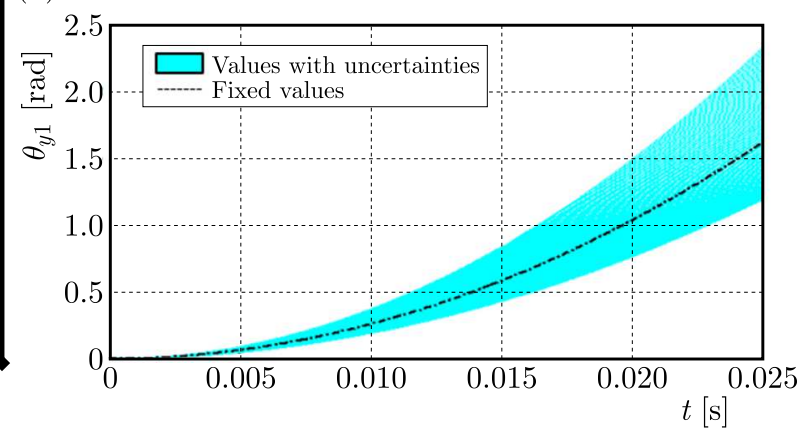

Fig. 8. Displacement of the gearbox input shaft with $3 \%$ of uncertainty on the module $m$

(a)

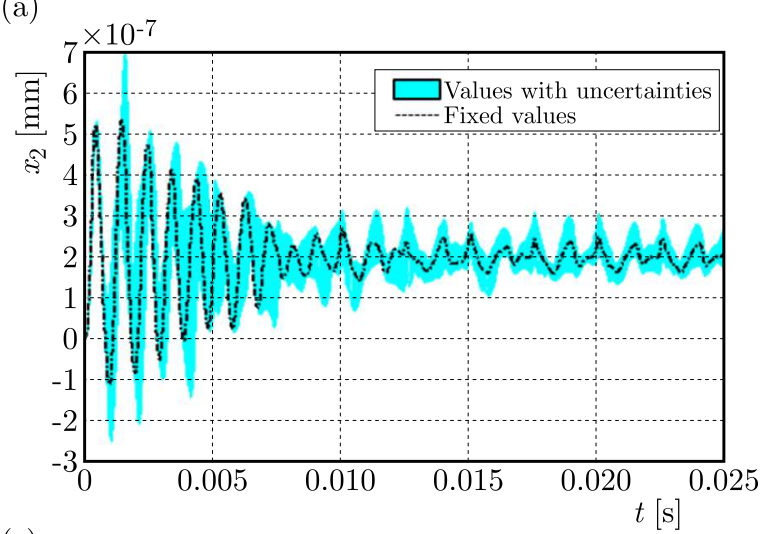

(c)

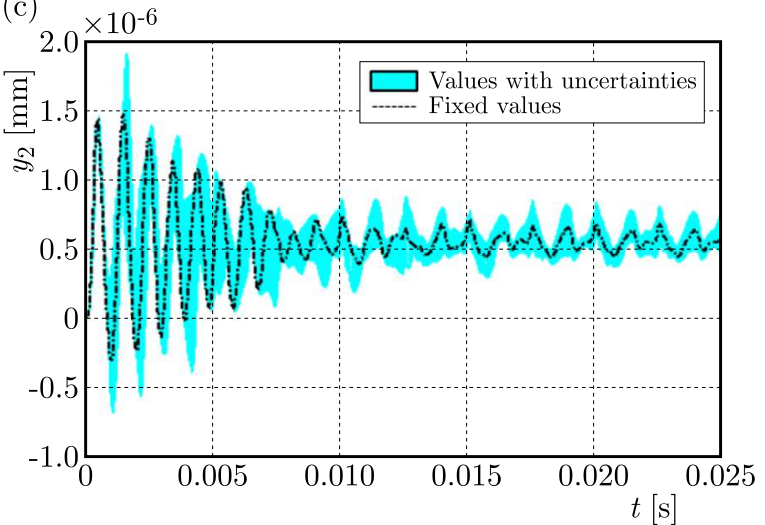

(b)

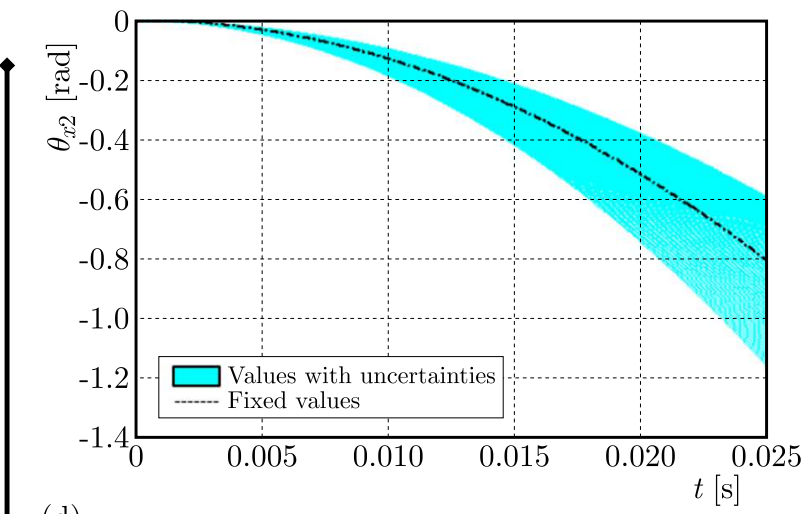

(d)

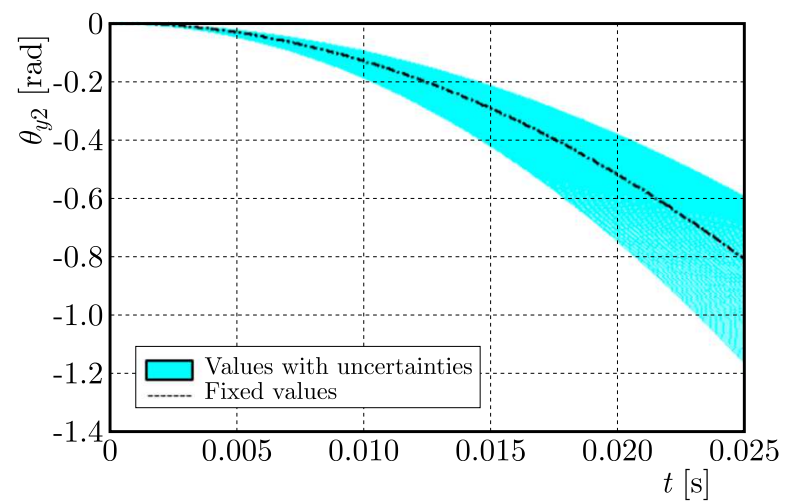

Fig. 9. Displacement of the gearbox output shaft with $3 \%$ of uncertainty on the module $m$ 
(a)

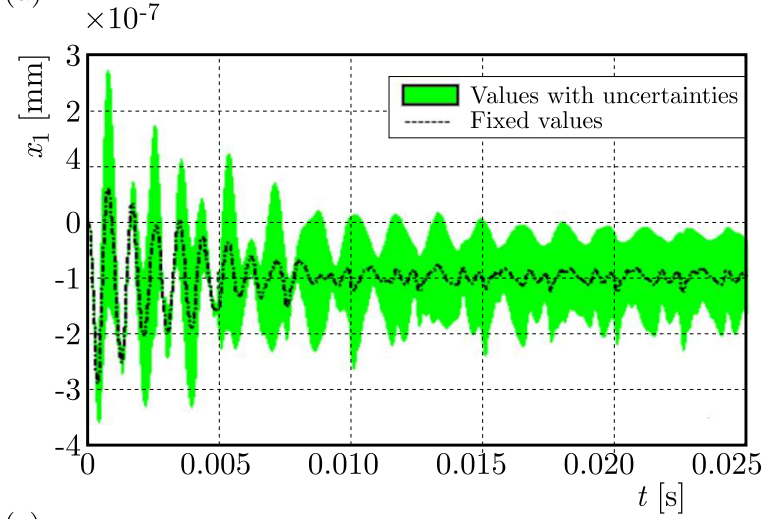

(c)

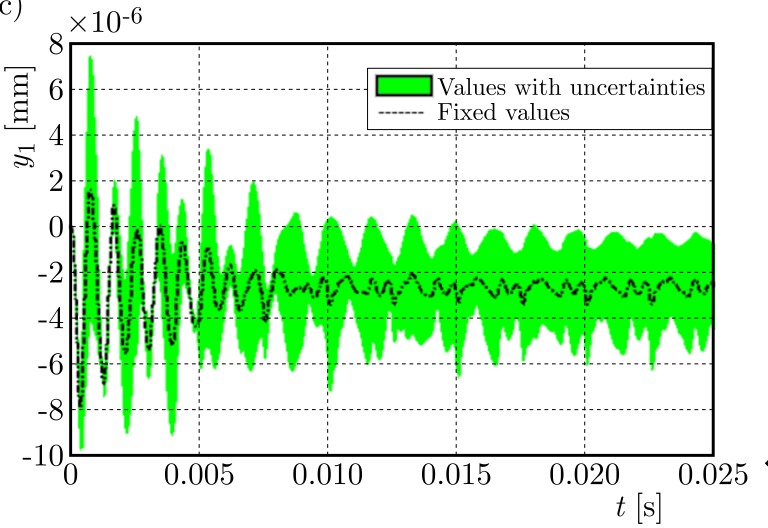

(b)

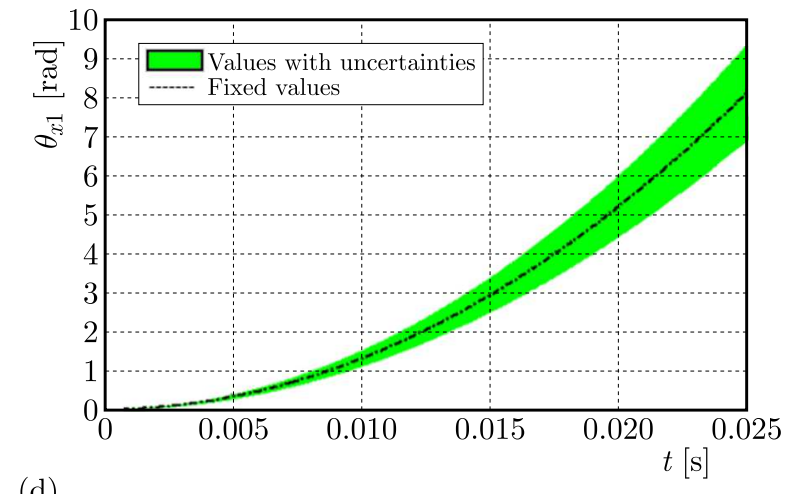

(d)

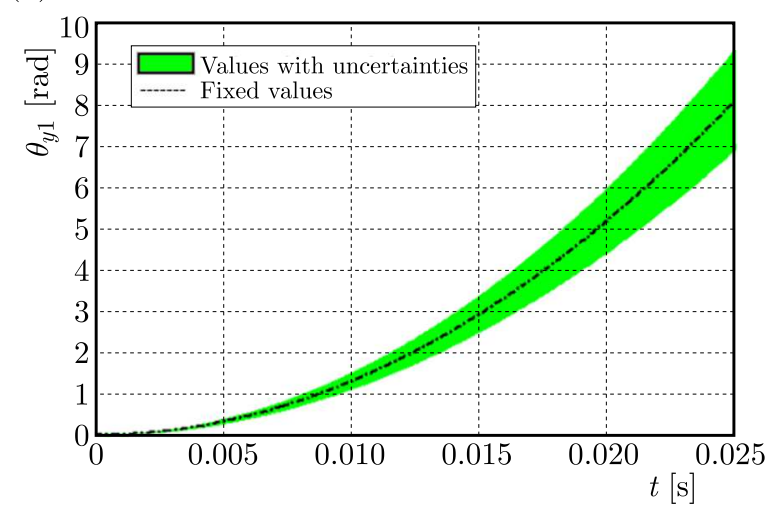

Fig. 10. Displacement of the gearbox input shaft with $3 \%$ of uncertainty on the receiver torque $c_{r}$

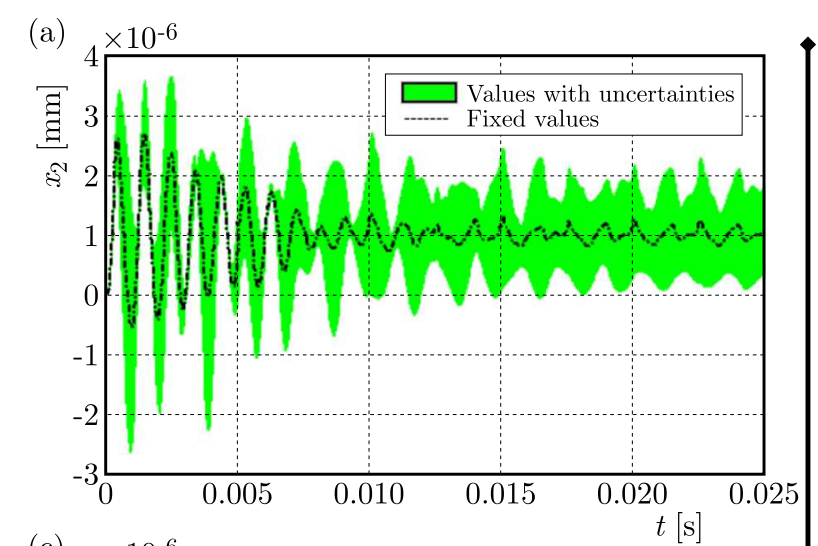

(c) $10 \times 10^{-6}$

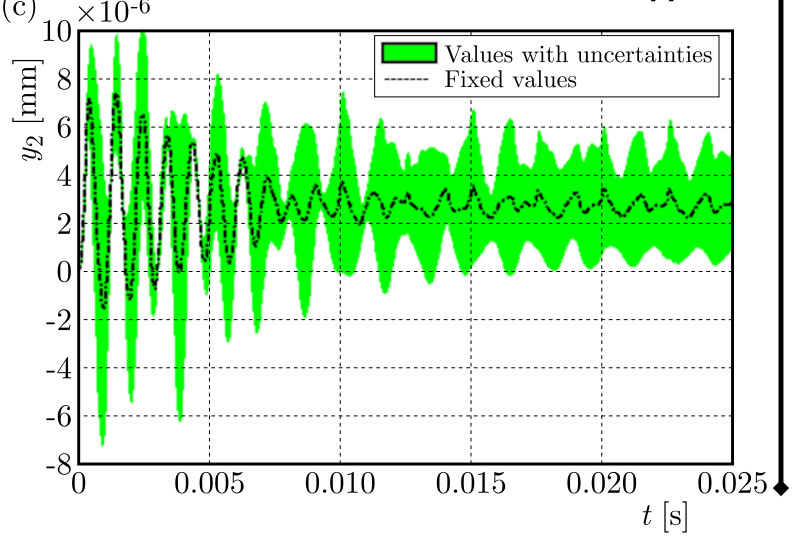

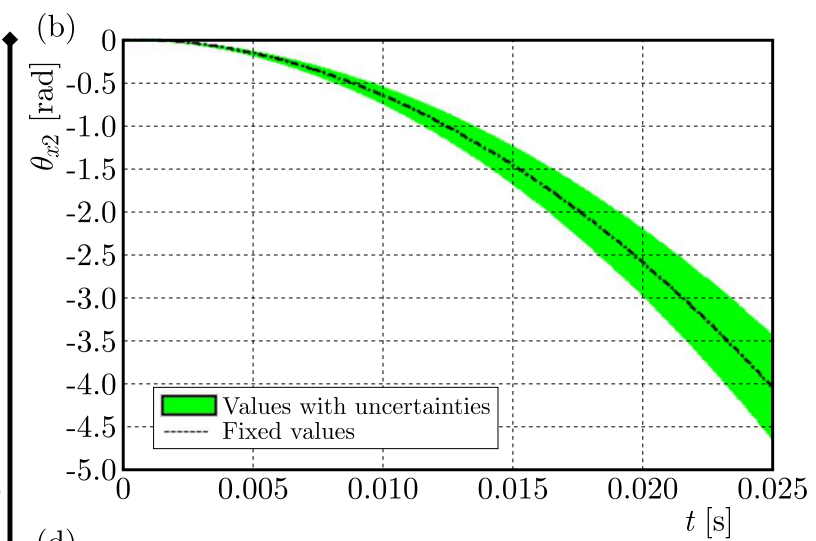

(d)

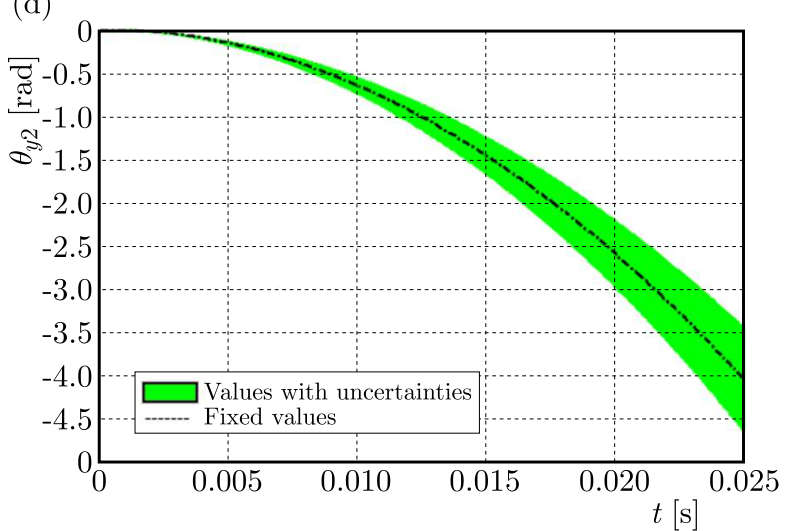

Fig. 11. Displacement of the gearbox output shaft with $3 \%$ of uncertainty on the receiver torque $c_{r}$ 
$\left(\theta_{x 1}, \theta_{x 2}, \theta_{y 1}, \theta_{y 2}\right)$ compared to the linear displacements $\left(x_{1}, x_{2}, y_{1}, y_{2}\right)$. On the other hand, the variation of $c_{r}$ acts mainly on the linear displacements.

The envelopes obtained for different displacements have the same paces as the curves obtained with fixed values. This shows the effectiveness of the applied method. These envelopes give a complete idea about functioning of the studied system. In addition, based on the results of simulations obtained, the designer can choose the right concept (solution) that better meets the performance requirements imposed, which facilitates the sizing of the system.

In conclusion, by coupling the interval calculation technique with the Newmark method, we obtained convergent results for an uncertainty rate of $3 \%$. In the following Section, a comparison using the Monte Carlo method is made to validate the simulations results (Vittal and Teboul, 2005).

\section{Validation with Monte Carlo}

To validate the results obtained previously, we used the Monte Carlo method. Simulation results of this method require a very long computation time. For this, we have limited our calculation only for the two displacements $x_{1}$ and $\theta_{x 1}$ in order to minimize the simulation time. Thus, 1000 random values (close to mean values of the variables $m$ and $c_{r}$ ) were chosen.

(a)

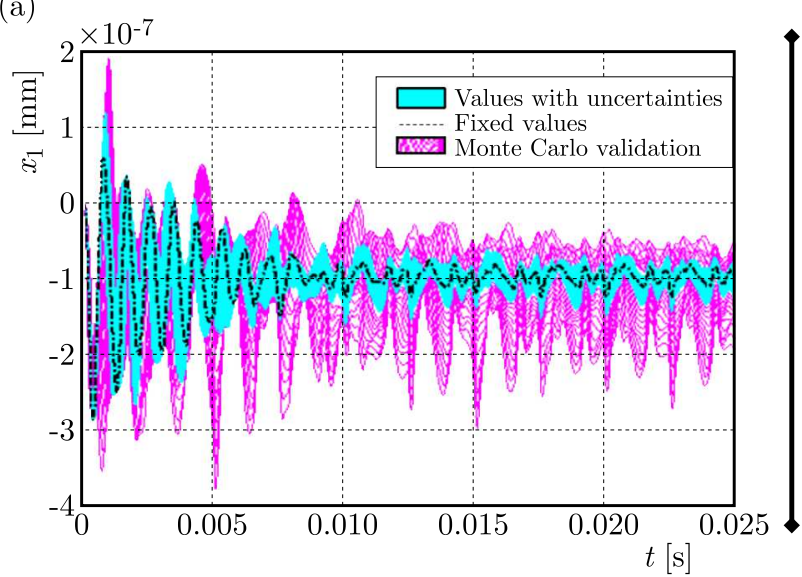

(b)

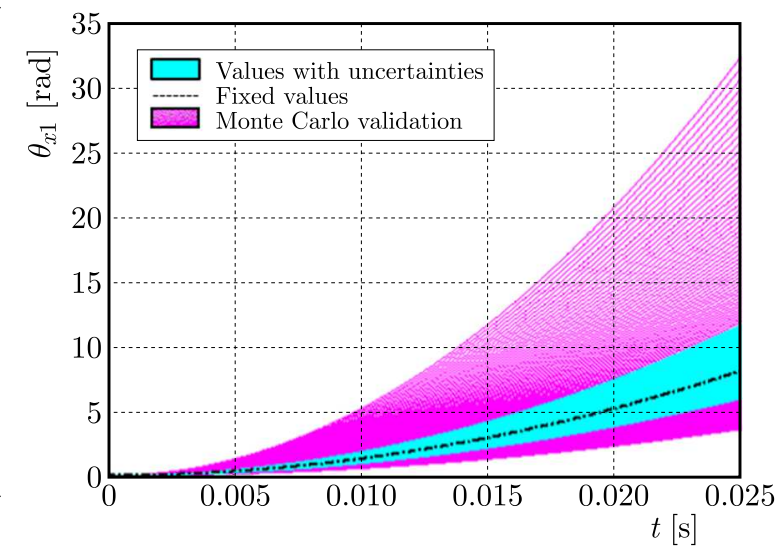

Fig. 12. Displacement of the gearbox input shaft with uncertainty on the module $m$ (using Monte Carlo simulation)

(a)

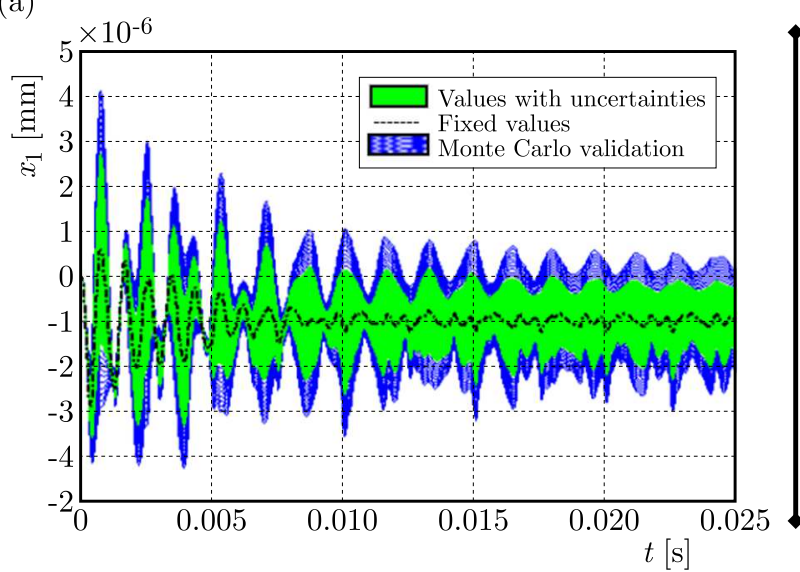

(b)

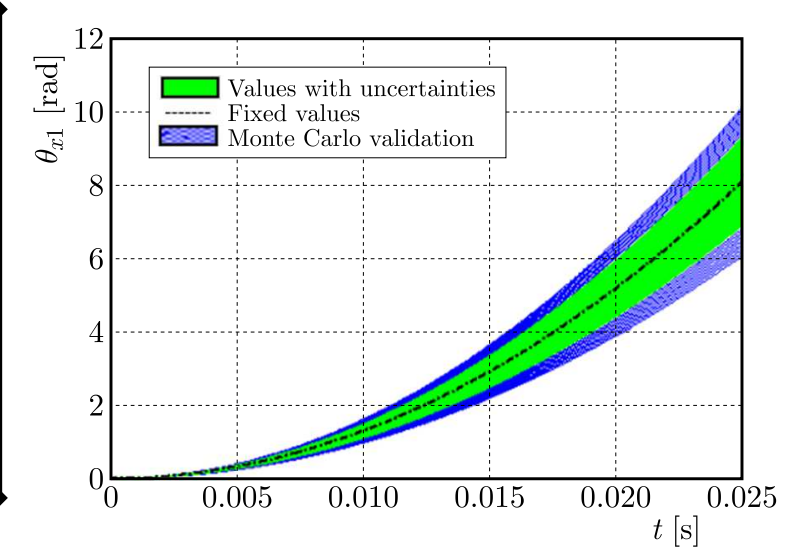

Fig. 13. Displacement of the gearbox input shaft with uncertainty on the receiver torque $c_{r}$ (using Monte Carlo simulation) 
The simulation results are shown in Figs. 12 and 13. The curves (in purple for $m$ and in blue for $c_{r}$ ) frame the envelopes obtained by the interval calculation technique. This shows that the results obtained previously are consistent with the actual dynamic behavior of the studied system.

\section{Conclusion}

In this study, the validity of the interval calculation technique coupled with the finite element method to simulate the static or dynamic behavior of a mechatronic system has been proven. Indeed, this type of calculation makes it possible to minimize the number of simulations, and consequently, to reduce the calculation time. Moreover, the proposed method offers a complete idea of the behavior of the system in the presence of uncertainties. This leads to a good sizing of the system to design. In the static case, the use of the intersection method allows one to limit the search space for different design parameters. This facilitates and helps the designer to choose the values that respect the required performance. In the dynamic case, the interval calculation coupling with the Newmark method provides not only a single evaluation of the behavior of the system, but rather an envelope of solutions. This gives a better description of the dynamic operation of the gearbox of a wind turbine with uncertain parameters. This coupling remains applicable for an uncertainty rate that does not exceed $3 \%$.

\section{References}

1. Alefeld G., Mayer G., 2000, Interval analysis: theory and applications, Journal of Computational and Applied Mathematics, 121, 421-464

2. Amendola G., Dimino i., Concilio A., Amoroso F., Pecora R., 2017, Preliminary design of an adaptive aileron for the next generation regional aircraft, Journal of Theoretical and Applied Mechanics, 55, 307-316

3. Chatri R., Khabou M.T., Barkallah M., Chaari F., Haddar M., 2016, Dynamic analysis of gearbox behaviour in milling process: non-stationary operations, Proceedings of the Institution of Mechanical Engineers, Part C: Journal of Mechanical Engineering Science, 230, 3372-3388

4. Dieterle W., 2005, Mechatronic systems: automotive applications and modern design methodologies, Annual Reviews in Control, 29, 273-277

5. FAng S.-E., Zhang Q.-H., Ren W.-X., 2015, An interval model updating strategy using interval response surface models, Mechanical Systems and Signal Processing, 60, 909-927

6. Faroughi S., Lee J., 2015, Analysis of tensegrity structures subject to dynamic loading using a Newmark approach, Journal of Building Engineering, 2, 1-8

7. Gilewski W., Peeczyński J., Rzeżuchowski T., Wąsowski J., 2015, Truss structures with uncertain parameters-geometrical interpretation of the solution based on properties of convex sets, Procedia Engineering, 111, 249-253

8. Guizani A., Hammadi M., Choley J.-Y., Soriano T., Abbes M.S., Haddar M., 2014, Multidisciplinary approach for optimizing mechatronic systems: application to the optimal design of an electric vehicle, 2014 IEEE/ASME International Conference on Advanced Intelligent Mechatronics, $56-61$

9. Навів M.K., 2007, Mechatronics - A unifying interdisciplinary and intelligent engineering science paradigm, IEEE Industrial Electronics Magazine, IEEE, 1, 12-24

10. Hammadi M., Choley J.-Y., Penas O., Riviere A., 2012, Multidisciplinary approach for modelling and optimization of Road Electric Vehicles in conceptual design level, Electrical Systems for Aircraft, Railway and Ship Propulsion, 1-6 
11. Hamza G., Choley J.-Y., Hammadi M., Riviere A., Barkallah M., Louati J., Haddar M., 2015, Pre-designing of a mechatronic system using an analytical approach with Dymola, Journal of Theoretical and Applied Mechanics, 53, 697-710

12. Hansen E., Sengupta S., 1981, Bounding solutions of systems of equations using interval analysis, BIT Numerical Mathematics, 21, 203-211

13. Henriot G., 1978, Traité théorique et pratique des engrenages, Tome II: Etude complète du matériel, 4 edit., Paris, Dunod Technique

14. Hughes T.J., 2012, The Finite Element Method: Linear Static and Dynamic Finite Element Analysis, Courier Corporation

15. Karnopp D.C., Margolis D.L., Rosenberg R.C., 2012, System Dynamics: Modeling, Simulation, and Control of Mechatronic Systems, John Wiley \& Sons

16. Ma Y., Liang Z., Chen M., Hong J., 2013, Interval analysis of rotor dynamic response with uncertain parameters, Journal of Sound and Vibration, 332, 3869-3880

17. Martins J.R., Lambe A.B., 2013, Multidisciplinary design optimization: a survey of architectures, AIAA Journal, 51, 2049-2075

18. Neumaier A., 1999, A simple derivation of the Hansen-Bliek-Rohn-Ning-Kearfott enclosure for linear interval equations, Reliable Computing, 5, 131-136

19. Nirmala T., Datta D., Kushwaha H., Ganesan K., 2013, The determinant of an interval matrix using Gaussian elimination method, International Journal of Pure and Applied Mathematics, 88, $15-34$

20. Trabelsi H., Yvars P.-A., Louati J., Haddar M., 2012, Effectiveness of an interval computation approach to the dynamic simulation of a Macpherson suspension system, 9th France-Japan and 7th Europe-Asia Congress on Mechatronics (MECATRONICS)/13th International Workshop on Research and Education in Mechatronics (REM), 78-85

21. Vittal S., Teboul M., 2005, Performance and reliability analysis of wind turbines using Monte Carlo methods based on system transport theory, 46th AIAA/ASME/ASCE/AHS/ASC Structures, Structural Dynamics and Materials Conference, 2218

22. Zienkiewicz O.C., TAylor R.L., 2005, The Finite Element Method for Solid and Structural Mechanics, Elsevier 